\title{
POWER HARVESTING AND TELEMETRY IN CMOS FOR IMPLANTED DEVICES
}

\author{
Christian Sauer, Milutin Stanacevic, Gert Cauwenberghs and Nitish Thakor
}

Johns Hopkins University, Baltimore

\begin{abstract}
Implanted sensors offer many advantages to those studying the behavior of the human body. Unfortunately, the need to power and communicate with devices often requires tradeoffs that compromise their usefulness. We describe a power harvesting and telemetry chip that allows operation without wires or batteries. The chip has been fabricated in $0.5 \mu \mathrm{m}$ CMOS. The chip is able to supply $2 \mathrm{~mA}$ at $3.3 \mathrm{~V}$ to associated sensors using inductive coupling. Tests reveal the characteristics of this chip under different loads and at different distances from the transmission coil. With the preliminary coils, functionality could be maintained with over $28 \mathrm{~mm}$ between the two coils. To evaluate the performance of the chip in conditions mimicking implantation in the body, water bearing colloids were introduced between the two coils. This resulted in a small loss of transfer efficiency, with no change in the behavior of the chip.
\end{abstract}

\section{INTRODUCTION}

Among the common applications of implanted microdevices are microelectrodes for neural and chemical electrical recording. In order to obtain enough power to amplify and record weak electrical signals, most electrodes are interfaced with wires connected through the skin. Unfortunately, this wiring or tethering limits the recording range in awake, behaving animals in chronic studies [1], [2].

Energy harvesting uses the external environment as a source of energy (e.g. temperature gradients, wind). For example, devices such as Smart Dust distributed networks make use of many different types of transducers, from solar power to vibration transducers [3]. Unfortunately, most of these types of energy gathering methods are unsuitable for a device implanted in the human body. A very promising alternative for implanted devices is RF power harvesting through inductive coupling [4]. In addition to gathering power, this technology can also be used to send data back to the base station, creating a two-way link [5], [6]. The principles of the technique are the same as those behind the increasingly common RFID (radio frequency identification) tags [7].

We have developed a power harvesting microchip based on this technique (Figure 1). The device is

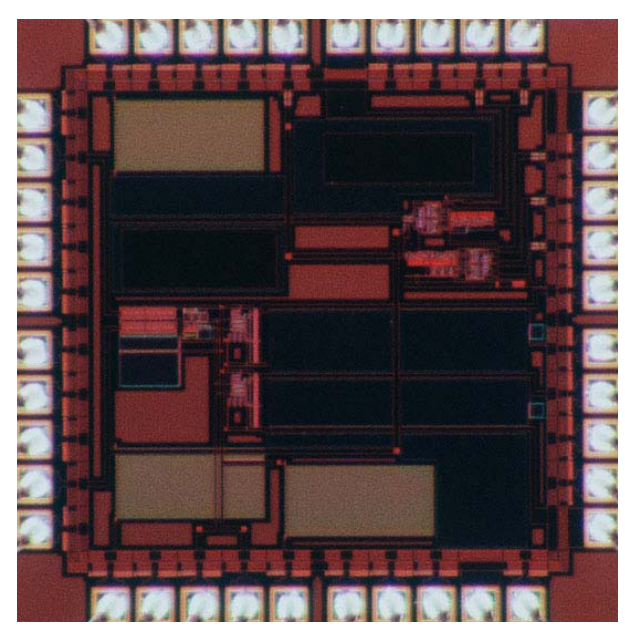

Figure 1. Photomicrograph of the fabricated chip.

designed to provide power, control signals, and a data link for an accompanied sensor to function. The chip can be utilized with many different types of sensors. Previous studies have shown that RF energy between 1 and $10 \mathrm{MHz}$ penetrates the body with minimum energy loss [8]. To take advantage of this biological characteristic we have designed our inductive link to operate at a frequency of $4 \mathrm{MHz}$. In this implementation, the chip supplies up to $2 \mathrm{~mA}$ at 3.3 Volts. In addition, it can be configured to supply various clocks and reference voltages necessary for the proper operation of the sensor. The chip is also designed to allow data transmission back to the module that broadcasts the power, removing any need for a physical connection.

\section{SYSTEM ARCHITECTURE}

As show in the system diagram (Figure 2), the chip can be broken down into several subunits: the rectifier, regulator, voltage bias, clock recovery, and data encoder. The transmission coil voltage is driven by a high efficiency class-E transmitter. The sinusoidal voltage on the receiver coil is dependant on the geometry of the coils. A full-wave rectifier followed by a low pass filter recovers a DC voltage. This voltage is dependant on the load and is not suitable for powering active circuitry. 


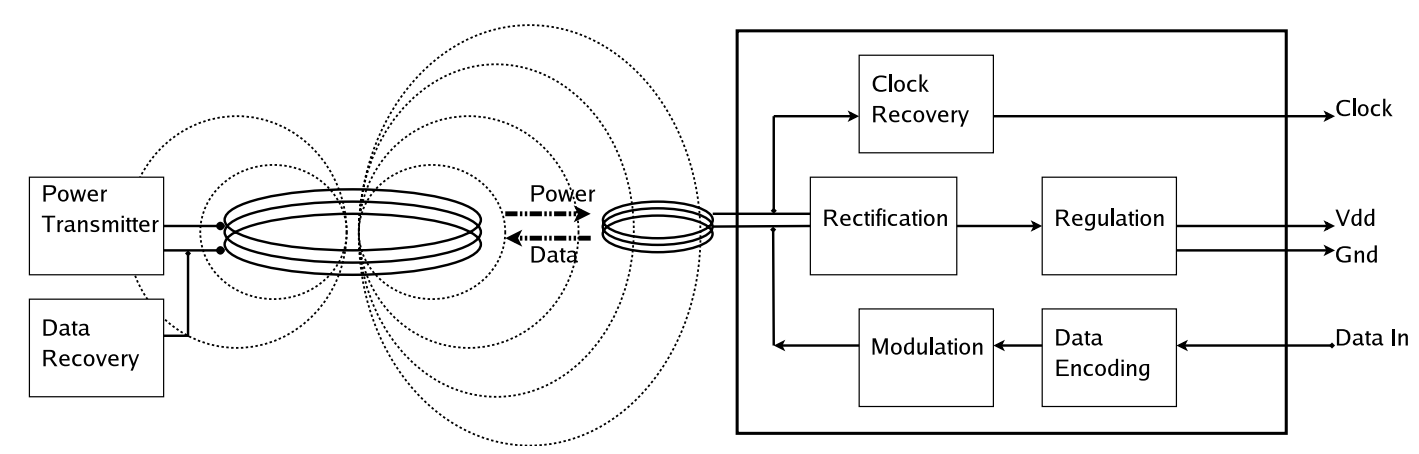

Figure 2. Block diagram of the system architecture

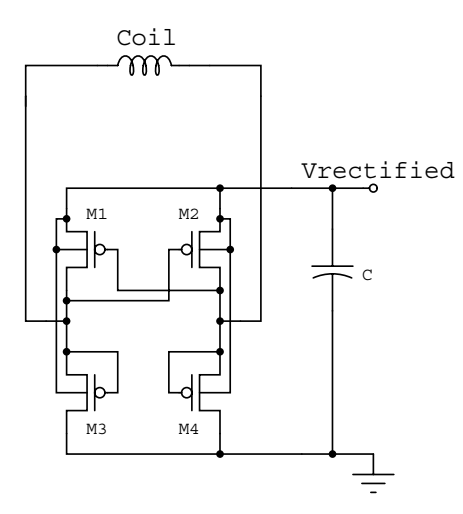

Figure 3. Circuit Diagram of the rectifier circuit

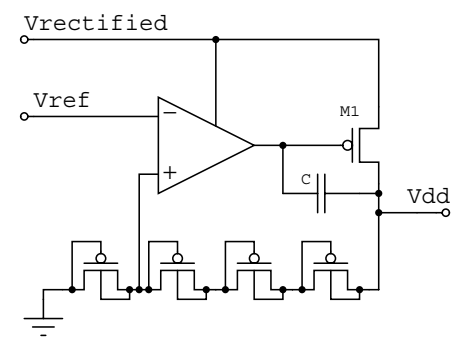

\section{Figure 4. Circuit Diagram of the regulator circuit}

A voltage regulator module reduces this voltage and holds it constant regardless of current draw. The value of the constant voltage is set using a voltage reference circuit; this block generates a voltage with no dependence on the voltage in the coil (as long as it is above a minimum value). We used separate analog and digital regulators on the microchip to avoid noise from the digital circuitry interfering with the more sensitive analog components. The entire analog system dissipates $35 \mu \mathrm{W}$.

A clock is recovered from the input sine wave. This allows the sensor to be synchronized with the transmitter and removes the need for an on-chip oscillator. Data (in NRZ format) is taken in for encoding and transmission to the base station. Transmission is accomplished through the same coil used to capture voltage. By modifying the impedance of this transponder coil it is possible to transmit data using very little energy. The change in coil impedance can be read out and decoded at the transmission station.

\subsection{Rectifier}

The rectifier circuit is a full wave rectifier composed of 4 PMOS transistors (Figure 3) [9]. BJTs and diodes, while somewhat feasible in a commercial CMOS process, have problems in this type of process and are avoided. When voltage on side A of the coil is high, M2 and M3 are shut off while M1 and M4 are turned on. This ties the low voltage side of the coil to ground while passing the high voltage. The situation is reversed when $\mathrm{B}$ is higher then $\mathrm{A}$.

The resulting output is passed though an $\mathrm{RC}$ filter with an external capacitor. By properly sizing the PMOS transistors the voltage drop is minimized and the rectified voltage is close to the RMS voltage of the received sine wave. For an output voltage of 3.3 Volts, a voltage amplitude of at least 7 Volts is required to be present on the coil. A standard CMOS process has a transistor gate break down voltage of 12 Volts, effectively setting an upper limit on the received voltage. The circuitry can be protected through the use of an off chip Zener diode, although in some cases this may be undesirable.

\subsection{Regulator}

The regulator circuit produces a steady $3.3 \mathrm{~V}$ output and can supply upwards of $2 \mathrm{~mA}$ (depending on the transmitted power). The circuit uses a transconductance amplifier to control the gate of a large PMOS transistor (M1 in Figure 4). Through negative feedback, the output voltage stabilized at a voltage proportional to the voltage reference. A feedback capacitor between the gate of the transistor and the output ensures system stability. Current draw of $100 \mu \mathrm{A}$ is necessary to keep the system operating in the proper regime.

\subsection{Voltage Reference}

The regulator requires a voltage reference to set the voltage output. The system is designed for implantation in the human body. A side effect is that the temperature of the implant will be held constant. 


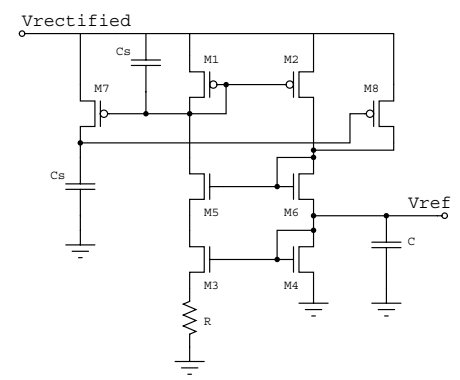

Figure 5. Circuit Diagram of the voltage reference.

This allows us to avoid the use of a band gap reference circuit (which would require BJTs). Instead, we have made use of a current bias circuit that allows a supply independent reference to be generated using only standard CMOS devices (Figure 5). A startup circuit (M7 and M8) moves the circuit out of its quiescent state. This circuit produces an $800 \mathrm{mV}$ reference voltage with a dependence of less then 1 percent on the supply [10].

\subsection{Clock Recovery}

A $4 \mathrm{MHz}$ clock is recovered from the sinusoidal waveform in the coil. A chain of inverters recovers a digital signal. This signal, synchronized with the base station, can be used for extracting data from the sensor. In addition, some sensors may require several different clock frequencies different from $4 \mathrm{MHz}$. These needs are provided for by stepping down the clock frequency. In the initial version of the chip a 1 $\mathrm{MHz}$ clock is provided, but can be augmented with other slower clocks.

\subsection{Data Encoding and Modulation}

Data is accepted from the sensor in NRZ format. The data is encoded in a modified Miller encoding scheme. For every logical one in the NRZ data stream a pulse is generated. The pulse width is controlled by an input clock for ease of testing. This clock could also be supplied by one of the clocks generated on chip. This encoding format uses two transitions for every one, but none for a zero. It requires little modulation of the coil. This is accomplished by turning on an NMOS transistor which connects a resistor between the coil and ground. This modulates the impedance of the coil, a change that can be read out on the transmission coil. With the current encoding scheme the amount of time that the resistor is in the circuit is minimized.

\section{MEASUREMENT RESULTS}

The chip has been fabricated in a $0.5 \mu \mathrm{m}$ bulk CMOS process through the MOSIS foundry. In order to test the chip with inductive coupling, we used a class-E transmitter was. This circuit produced a high power RF signal at $4 \mathrm{MHz}$, using an antenna coil $5 \mathrm{~cm}$ in diameter. A similar but smaller coil $(2 \mathrm{~cm}$ in diameter) was used to harvest the broadcast energy.

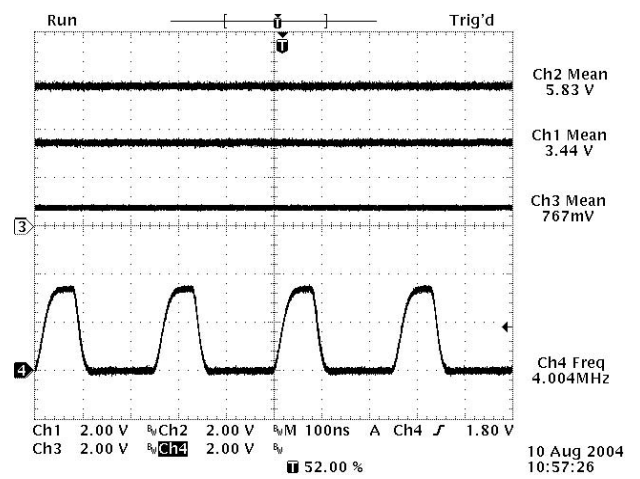

Figure 6. Oscilloscope trace of the microchip waveforms

The two components were realized on different prototype boards, with different ground planes. Figure 6 illustrates the basic waveforms of the chip during operation.

\subsection{Air Coupling at Various Distances}

To test the coupling and determine the maximum power transfer the transmitter and receiver coils were placed close together and moved apart. As shown in Figure 7 , distances between $10 \mathrm{~mm}$ and $100 \mathrm{~mm}$ were tested. The chip was loaded such that it would produce $0.7 \mathrm{~mA}$ when the voltage was enough to operate the regulator. With this load the chip was able to function with up to $28 \mathrm{~mm}$ between the two coils.

\subsection{Load Regulation}

The amount of current the chip could source before the regulator became inoperative was also tested (Figure 8). The maximum current sourced depends on the distance between the two coils. The amount of voltage change until the drop off point was constant across all distances tested.

\subsection{Coupling and Interference}

The previous tests examine the characteristics of the system when only air exists between the two coils. However, as the device is intended to be implanted, this assumption is unrealistic. Biological tissue will obviously have some effect on the coupling between the two coils. While truly recreating biological tissue was beyond the scope of this investigation, we made use of the fact that the main component of the human body is water. We added water bearing colloid samples between the two coils. This allowed us to control the thickness of the sample without an elaborate setup. The results are shown in Figure 9. The distance between the coils was held constant at $25 \mathrm{~mm}$ and the root mean square of the recovered voltage measured. The plot shows the percentage change from the base value (air coupling at $25 \mathrm{~mm}$ ) when samples of different thickness are interposed between the two coils. 


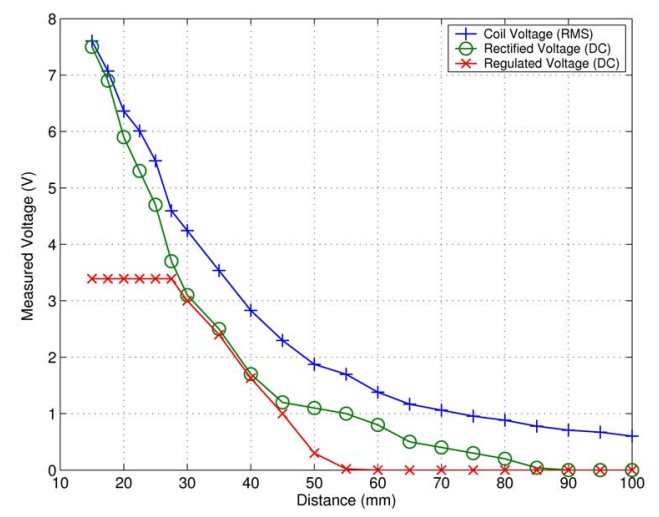

Figure 7. Air coupling at various distances
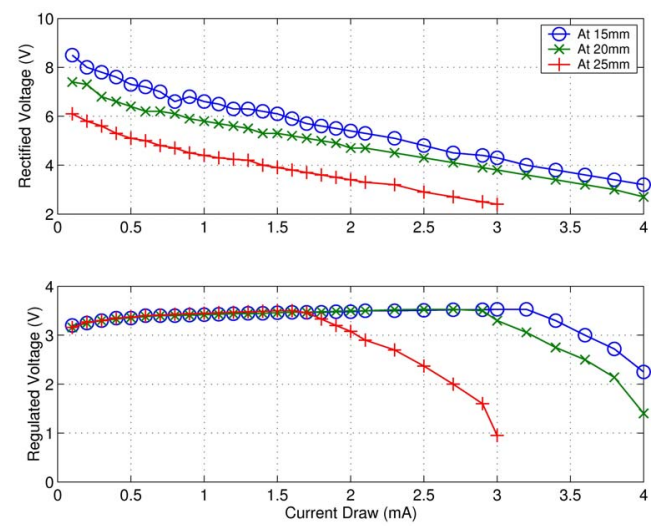

Figure 8. Load regulation

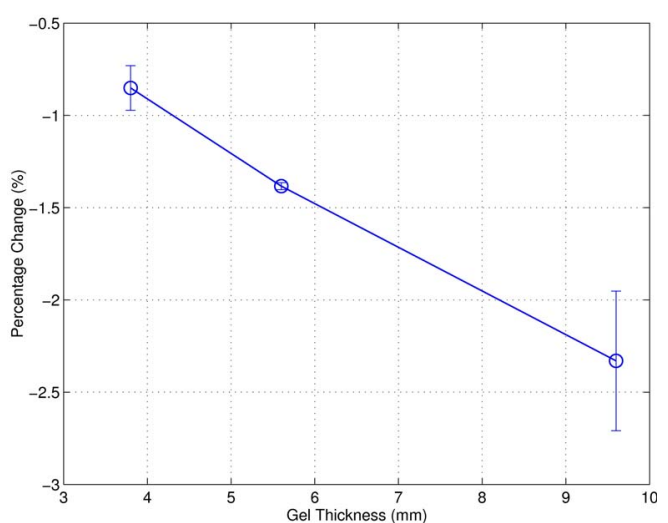

Figure 9. Coupling losses due to medium layers

\section{DISCUSSION}

We have presented the design and initial test results for a generic chip that can be used to power and interface with an implanted sensor. The chip has dual voltage regulators that can be used to supply both digital and analog sources. Both voltages were designed to be $3.3 \mathrm{~V}$. In tests these voltages were actually measured to be 3.4 and $3.5 \mathrm{~V}$. This mismatch is probably due to transistor sizing in the layout. In the next version we plan to eliminate this dependence. In addition, we intend to study further the effect of interference between the two coils. Coil sizing is expected to play a major role in this dependency and we are currently performing FEM analysis of the intervening conductive media to determine the optimal coil sizes.

\section{ACKNOWLEDGMENTS}

This work was supported in part by the NIH grant MH62444. Chips were fabricated with the MOSIS foundry.

\section{REFERENCES}

[1] A. Belayev, I. Saul, Y. Liu, W. Zhao, M. D. Ginsberg, M. A. Valdes, R. Busto, and L. Belayev, "Enriched environment delays the onset of hippocampal damage after global cerebral ischemia in rats," Brain Res., 964, 1, pp. 121-127, 2003.

[2] A. Risedal, B. Mattsson, P. Dahlqvist, C. Nordborg, T. Olsson, and B. B. Johansson, "Environmental influences on functional outcome after a cortical infarct in the rat," Brain. Res. Bull., 58, 3, pp. 315-321, 2002.

[3] B. Warneke, B. Atwood, and K. S. Pister, "Preliminary smart dust mote," Hot Chips, 12, 1, 2000.

[4] W. J. Heetderks, "RF powering of millimeterand submillimeter-sized neural prosthetic implants," IEEE Trans. Biomed. Eng., 33, 5, pp. 323-327, 1988.

[5] P. Irazoqui-Pastor, I. Mody, and J. W. Judy, "In-vivo EEG recording using a wireless implantable neural transceiver," IEEE EMBS Conference on Neural Engineering, 1, pp. 622-625, 2003.

[6] T. Akin, K. Najafi, and R. M. Bradley, "A wireless implantable multichannel digital neural recording system for a micromachined sieve electrode," IEEE Journal of Solid-State Circuits, 33, 1, pp. 109-118, 1998.

[7] P. Vaillancourt, A. Djemouai, J. F. Harvey, and M. Sawan, "EM radiation behavior upon biological tissues in a radio-frequency power transfer link for a cortical visual implant," IEEE EMBS Conference, 6, 1, pp. 2499-2502, 1997.

[8] K. Finkenzeller, RFID Handbook, J. Wiley and Sons, England, 2003.

[9] P. Rakers, L. Connell, T. Collins, and D. Russell, "Secure Contactless Smartcard ASIC with DPA Protection", IEEE Journal of Solid-State Circuits, 36, 3, pp. 559-565, 2001.

[10] T. Delbruck and A. van Schaik, "Bias current generators with wide dynamic range", ISCAS 2004, 1, pp. I-337 - I-340, 2004. 